\title{
Single skeletal muscle fiber behavior after a quick stretch in young and older men: a possible explanation of the relative preservation of eccentric force in old age
}

\author{
Julien Ochala • David D. Dorer • Walter R. Frontera • \\ Lisa S. Krivickas
}

Published online: 5 October 2006

(C) Springer-Verlag 2006

The online version of the original article can be found at http://dx.doi. org/10.1007/s00424-006-0065-6.

J. Ochala $\cdot$ W. R. Frontera $\cdot$ L. S. Krivickas

Muscle Cell Physiology Laboratory,

Department of Physical Medicine and Rehabilitation,

Spaulding Rehabilitation Hospital and Harvard Medical School,

Boston, MA, USA

J. Ochala

Equipe INSERM-ERM 207 Motricité-Plasticité,

Faculté des Sciences du Sport, Université de Bourgogne,

Dijon, France

D. D. Dorer

Biostatistics Center, Massachusetts General Hospital,

Boston, MA, USA

J. Ochala $(\bowtie)$

Department of Neuroscience, Clinical Neurophysiology,

University Hospital,

Entrance 85, room 6511,

Uppsala 75185 , Sweden

e-mail: julien.ochala@neurofys.uu.se
Pflügers Arch—Eur J Physiol (2006) 452: 464-470

In the "Discussion" section, the beginning of the paragraph "Force responses to stretch with aging" should read:

During phase $1, \Delta T_{1}$ was lower in older men in both type I and IIa fibers. Single muscle fibers appear to be more resistant to elastic distortion in older men. The decrease in $\Delta T_{1}$ can only be caused by a lower total elastic distortion of the attached cross-bridges and myofilaments, i.e., the fibers are less compliant [22]. This lower compliance can be due to either a lower number of strongly bound actin-myosin cross-bridges with the same compliance per cross-bridge and myofilaments, or the same number of strongly bound actin-myosin crossbridges with a lower compliance per cross-bridge and myofilaments. 